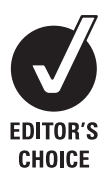

Department of Ophthalmology, Institute of Clinical Medicine, University of Tsukuba, Ibaraki, Japan

Correspondence to: Dr T Oshika, Department of Ophthalmology, Institute of Clinical Medicine, University of Tsukuba, 1-1-1 Tennoudai, Tsukuba, Ibaraki 305-8575, Japan; toshika@md.tsukuba.ac. jp

Accepted 7 May 2009 Published Online First 30 June 2009

\title{
Vision-related quality of life and visual function in patients undergoing vitrectomy, gas tamponade and cataract surgery for macular hole
}

\author{
S Fukuda, F Okamoto, M Yuasa, T Kunikata, Y Okamoto, T Hiraoka, T Oshika
}

\begin{abstract}
Aim: To evaluate the relationship between vision-related quality of life (VR-0OL) and visual function in patients undergoing vitrectomy, gas tamponade and cataract surgery for macular hole (MH).
\end{abstract}

Methods: The 25-item National Eye Institute Visual Function Questionnaire (VFO-25) was self-administered by 32 patients with $\mathrm{MH}$ (age 66.2 (SD 5.4) years) preoperatively and at 3 months postoperatively. Clinical data were collected, including logarithm of minimum angle of resolution (logMAR) best corrected visual acuity (BCVA), severity of metamorphopsia and letter contrast sensitivity. The severity of metamorphopsia was evaluated by the M-Charts. MH index was measured using optical coherence tomography. The presence and severity of cataract were graded using the Lens Opacities Classification System III reference standards. Multiple regression analysis was performed to investigate the relationship between various explanatory variables and VFO-25 questionnaire scores. Explanatory variables tested were the severity of metamorphopsia, visual acuity, letter contrast sensitivity, MH index and grade of cataract.

Results: Vitrectomy for MH significantly improved VFO25 composite score as well as subscale scores, including general vision, near activities, distance activities, social functioning, mental health and dependency $(p<0.05$, Wilcoxon signed-rank test). Multiple regression analysis revealed that, both preoperatively and postoperatively, the severity of metamorphopsia had a significant correlation with VFO-25 composite score $(p<0.05)$, whereas other explanatory variables did not. In addition, changes in the severity of metamorphopsia was the single variable that was significantly related to changes in VFO-25 composite score $(p<0.01)$.

Conclusion: Vitrectomy for $\mathrm{MH}$ significantly improved VR-0OL. The severity of metamorphopsia was significantly associated with both preoperative and postoperative VRQOL.

Visual acuity improves in many patients after successful closure of macular hole $(\mathrm{MH})$ by vitrectomy, ${ }^{12}$ whereas some patients still complain of remaining metamorphopsia after surgery. Metamorphopsia is one of the most common conditions that cause deterioration in visual functions in patients with macular diseases. Relatively little attention has been paid, however, to changes in visual functions, including metamorphopsia, caused by surgery and their relationship with vision-related quality of life (VR-QOL).

Recent studies using the 25-item National Eye Institute Visual Function Questionnaire (VFQ-25) have reported that vitrectomy for $\mathrm{MH}$ appears to have a beneficial effect on patients' subjective perception of quality of life..$^{3-5}$ Tranos et al demonstrated that VFO-25 score after $\mathrm{MH}$ surgery was significantly correlated with the severity of postoperative metamorphopsia. ${ }^{4}$ On the other hand, preoperative and surgery-induced changes in VFO-25 composite score did not correlate with the preoperative severity of metamorphopsia or changes in the severity. ${ }^{4}$ They evaluated the severity of metamorphopsia using the Amsler chart, which has been widely used to detect and evaluate metamorphopsia in patients with macular diseases. ${ }^{67}$ With the Amsler chart, however, it is difficult to quantify the severity of metamorphopsia because the patients have to self-describe the degree of image distortion. Assessment with the M-Charts (Inami Co., Tokyo, Japan) can easily and quantitatively evaluate the degree of metamorphopsia associated with macular diseases, as the patients only have to state whether the line appears to be distorted or not. ${ }^{8}$ We conducted the current prospective study to evaluate the relationship between VR-QOL and visual function, including metamorphopsia, using the $\mathrm{M}$-Charts in patients undergoing vitrectomy for $\mathrm{MH}$.

\section{PATIENTS AND METHODS}

The current prospective, consecutive case series included 32 eyes of 32 patients with $\mathrm{MH}$ (13 men and 19 women) who underwent pars plana vitrectomy at Tsukuba University Hospital between June 2006 and February 2007. Patients with other ocular diseases, except for mild refractive error, were excluded. There were no significant ocular pathologies in the fellow eyes of the patients. Logarithm of minimum angle of resolution (logMAR) best corrected visual acuity (BCVA), the severity of metamorphopsia and letter contrast sensitivity were recorded preoperatively and at 3 months postoperatively.

The severity of metamorphopsia was evaluated by the $\mathrm{M}$-Charts. The $\mathrm{M}$-Charts consist of 19 dotted lines with dot intervals ranging from $0.2^{\circ}$ to $2.0^{\circ}$ of visual angle. If the straight line is substituted with a dotted line and the dot interval is changed from fine to coarse, the distortion of the line decreases with the increasing dot interval, until finally the dotted line appears straight. ${ }^{8}$

Letter contrast sensitivity was measured using the CSV-1000LV chart (Vector Vision, Columbus, Ohio, USA). Measurements with optical coherence tomography (OCT; Stratus OCT 3000, Carl Zeiss Ophthalmic Systems-Humphrey Division, Dublin, California, USA) were carried out preoperatively. 
Three $\mathrm{MH}$ parameters $(\mathrm{MH}$ base diameter, $\mathrm{MH}$ diameter and retinal thickness around the $\mathrm{MH}$ ) were defined and the $\mathrm{MH}$ index (MHI) was calculated according to the methods of Kusuhara et al. ${ }^{10}$ For standardisation purposes, several OCT images were recorded on each occasion. The presence and severity of cataract were graded using the Lens Opacities Classification System III (LOCS III) reference standards. ${ }^{11}$

The patients were requested to answer VFQ-25 preoperatively and at 3 months postoperatively to assess VR-QOL. ${ }^{12}$ The research staff explained the questionnaire to the patients, and gave verbal instructions and provided assistance when required. The completed questionnaires were reviewed for missing data by the research staff. Prior to surgery, all missing data were added by the patients themselves. The VFO-25 comprises 25 items wherein patients are expected to assess the level of difficulty of particular visual symptoms or day-to-day activities. Each item is assigned to one of the 12 subscales: general health, general vision, ocular pain, near activities, distance activities, social functioning, mental health, role difficulties, dependency, driving, colour vision and peripheral vision. The subscales are scored on a 0 to 100 point scale, where 100 indicates the highest possible function or the minimal subjective impairment. The VFQ-25 composite score is calculated as the unweighted average response to all items, excluding questions regarding general health. The VFO-25 used in this study was a Japanese version, with modifications to suit the Japanese culture and way of life. The modified NEI VFO-25 questionnaire has been assessed for reliability and validity, and it has been proven to accurately measure VR-QOL in Japanese individuals. ${ }^{13}$

Surgeries were performed by an experienced vitreoretinal surgeon (F O), using standard cataract surgery and pars plana vitrectomy with 20 gauge probes, peeling of the inner limiting membrane with subsequent instillation of a gas tamponade (20\% sulphur hexafluoride; $\mathrm{SF}_{6}$ ) and postoperative instructions to lie face down for 1 week. Indocyanine green was used to peel the inner limiting membrane. Subjects were followed up for at least 3 months after surgery and any postoperative complications were documented.

Mean scores and standard deviations were calculated for VFO-25 composite and subscale scores, visual acuity, severity of metamorphopsia, contrast sensitivity, MHI and LOCS III index. The significance of the differences between preoperative and postoperative values was assessed with the Wilcoxon signedrank test. Preoperatively and postoperatively, multiple regression analysis was performed to investigate the relationship between various explanatory variables and VFQ-25 questionnaire scores. Variables tested were visual acuity, severity of metamorphopsia, letter contrast sensitivity, MHI and grade of cataract (LOCS III index). The relationship between changes in VFO-25 composite score and changes in above-mentioned explanatory variables was also evaluated. All statistical tests were considered to be significant if $p<0.05$. Analyses were carried out using StatView (version 5.0; SAS Institute, Cary, North Carolina, USA). A prestudy power calculation using a significance level of $5 \%(\alpha)$ and a power of $80 \%(1-\beta)$ revealed that a sample size of 27 eyes would be required to detect a mean VFQ-25 composite score difference of 6 points.

\section{RESULTS}

Table 1 summarises the preoperative characteristics of patients. By the initial surgery, $\mathrm{MH}$ was successfully closed in all eyes. No significant intraoperative and postoperative complications were observed, such as retinal detachment, choroidal detachment, subretinal haemorrhage, cystoid macular oedema or infection.
Table 1 Preoperative characteristics of patients with macular hole

\begin{tabular}{lc}
\hline Variable & Value \\
\hline Age (years) & $66.2(5.4)$ \\
Male/female (n/n) & $13 / 19$ \\
LogMAR BCVA in worse-seeing eye (operated eye) & $0.76(0.47)$ \\
LogMAR BCVA in better-seeing eye (fellow eye) & $0.04(0.26)$ \\
Stage of macular hole (II/III/IV) (n) & $7 / 16 / 9$ \\
LOCS III (scale 0.1-5.9) & \\
$\quad$ Nuclear colour & $1.20(0.3)$ \\
Nuclear opalescence & $0.60(0.8)$ \\
Cortical opacity & $0.86(1.0)$ \\
Posterior subcapsular opacity & $0.27(0.4)$ \\
Severity of metamorphopsia & $0.77(0.55)$ \\
Letter contrast sensitivity & $15.3(8.8)$ \\
Macular hole index & $0.81(0.56)$ \\
\hline
\end{tabular}

\section{Values are mean (SD).}

BCVA, best corrected visual acuity; LOCS III, Lens Opacities Classification System III; LogMAR, logarithm of minimum angle of resolution.

In six patients, intraocular pressure elevated to $25 \mathrm{mmHg}$ or higher, but responded promptly to antihypertensive therapy.

All patients underwent $\mathrm{MH}$ surgery on their worse-seeing eyes. The surgery significantly improved the metamorphopsia score from 0.77 (SD 0.55) to 0.45 (SD 0.44) ( $<<0.005$, Wilcoxon signed-rank test), logMAR BCVA from 0.76 (SD 0.47) to 0.48 (SD 0.35) $(p<0.0001)$, and letter contrast sensitivity from 15.3 (SD 8.8) to 19.5 (SD 8.2) $(\mathrm{p}<0.005)$. Metamorphopsia was significantly improved by surgery, but did not resolve completely at 3 months after surgery.

The results of the pre- and postoperative VFQ-25 questionnaire are shown in table 2 . Vitrectomy significantly improved VFQ-25 composite score $(p<0.005)$, as well as subscales such as general vision $(p<0.0005)$, near activities $(p<0.001)$, distance activities $(p<0.05)$, social functioning $(p<0.01)$, mental health $(\mathrm{p}<0.05)$ and dependency $(\mathrm{p}<0.01)$.

Table 3 summarises the results of multiple regression analysis on the relationship between VFO-25 composite score and several explanatory variables, including visual function, $\mathrm{MH}$ morphology and grade of cataract. Both preoperatively and postoperatively, the severity of metamorphopsia exhibited significant positive correlation with VFQ-25 composite score $(p<0.05)$, whereas other explanatory variables showed no relationship with VFQ-25 composite score. Similarly, changes in the severity of metamorphopsia were significantly associated with the changes in VFO-25 composite score $(p<0.01$, fig 1$)$, but changes in other variables were not, including logMAR BCVA (fig 2) and letter contrast sensitivity (fig 3).

\section{DISCUSSION}

As shown in the results, vitrectomy for $\mathrm{MH}$ significantly improved VFQ-25 composite score and subscale scores including general vision, near activities, distance activities, social functioning, mental health and dependency. This observation is consistent with the results of previous studies that also investigated VR-QOL in patients with $\mathrm{MH}^{3}{ }^{3-5}$

In the current study, multiple regression analysis revealed that the severity of metamorphopsia was significantly associated with VFQ-25 composite score, both preoperatively and postoperatively, while other variables were not related, such as visual acuity, letter contrast sensitivity, MHI and grade of cataract (LOCS III index). Further, changes in the severity of metamorphopsia were significantly correlated with changes in VFQ-25 composite score. These findings are not consistent with 
Table 2 The National Eye Institute 25-item Visual Function Questionnaire (VFQ-25) composite and 12 subscale scores in patients with macular hole

\begin{tabular}{llll}
\hline & \multicolumn{2}{l}{ VFQ-25 scores } & \\
\cline { 2 - 4 } VF0-25 scale & Before surgery & After surgery & p Value \\
\hline Composite score & $70.5(17.1)$ & $77.4(11.2)$ & $0.002^{*}$ \\
General health & $49.2(14.6)$ & $50.0(14.2)$ & 0.763 \\
General vision & $53.7(15.6)$ & $68.1(13.3)$ & $0.001^{*}$ \\
Ocular pain & $81.6(16.7)$ & $84.3(14.9)$ & 0.240 \\
Near activities & $58.6(19.2)$ & $70.1(16.8)$ & $0.001^{*}$ \\
Distance activities & $62.2(19.0)$ & $69.7(16.1)$ & $0.019^{*}$ \\
Social functioning & $77.4(15.2)$ & $82.8(13.4)$ & $0.007^{*}$ \\
Mental health & $66.0(21.1)$ & $75.4(12.7)$ & $0.015^{*}$ \\
Role difficulties & $69.9(21.7)$ & $76.2(21.6)$ & 0.053 \\
Dependency & $82.0(17.7)$ & $89.6(11.0)$ & $0.008^{*}$ \\
Driving & $72.2(18.4)$ & $76.7(15.2)$ & 0.667 \\
Colour vision & $85.1(14.0)$ & $86.7(16.8)$ & 0.617 \\
Peripheral vision & $66.4(17.5)$ & $73.0(18.0)$ & 0.065 \\
\hline
\end{tabular}

Values are mean (SD).

${ }^{*}$ Significantly improved by surgery (Wilcoxon signed-rank test).

the results of a previous study. ${ }^{4}$ Tranos et al investigated VR$\mathrm{QOL}$ in patients with $\mathrm{MH}$ and reported that postoperative VFO-25 scores were significantly correlated with the severity of postoperative metamorphopsia recorded by measuring the number of squares representing distortion on the Amsler chart. However, preoperative and surgery-induced changes in VFO-25 composite score were not associated with preoperative and changes in the severity of metamorphopsia. ${ }^{4}$ The discrepancy between our study and the previous studies may be attributable to the different methodology used to evaluate metamorphopsia. In previous studies, the severity of metamorphopsia was graded using the Amsler charts, which have been widely used to detect metamorphopsia in macular diseases. ${ }^{67}$ With the Amsler chart, however, precise and reproducible assessment of metamorphopsia is difficult because patients have to self-describe the degree of image distortion. On the other hand, the M-Charts can evaluate the degree of metamorphopsia quantitatively since patients need simply to answer whether the dotted line is distorted or not. With this chart, the degree of metamorphopsia is graded quantitatively from 0 to $2.0 .^{89}$ It has been reported that the metamorphopsia score recorded by the M-charts increased depending on the severity of membrane proliferation classified by scanning laser ophthalmoscope images in patients with epiretinal membrane. ${ }^{8}$ Another study demonstrated that there was a significant positive correlation between the degree of retinal contraction obtained by using image-analysis software and metamorphopsia score quantified by the $\mathrm{M}$-charts in patients with idiopathic epiretinal membrane. ${ }^{9}$

Preoperative, postoperative and surgery-induced changes in VFO-25 scores did not correlate with visual acuity in both the better- and worse-seeing (operated) eyes. Miskala et al measured VFO-25 in patients with subfoveal choroidal neovascularisation caused by age-related macular degeneration, and demonstrated that VFQ-25 subscale scores were significantly correlated with visual acuity in the better-seeing eyes, but not with visual acuity in the worse-seeing eyes. ${ }^{14}$ In patients with unilateral central retinal vein occlusion, Deramo et al reported that VFQ25 responses were significantly correlated with visual acuity in the better-seeing eyes. ${ }^{15}$ In patients with early glaucoma with similar visual acuity bilaterally, Hyman et al demonstrated that VFO-25 responses were significantly correlated with visual acuity in both the better- and worse-seeing eyes. ${ }^{16}$ Conversely,

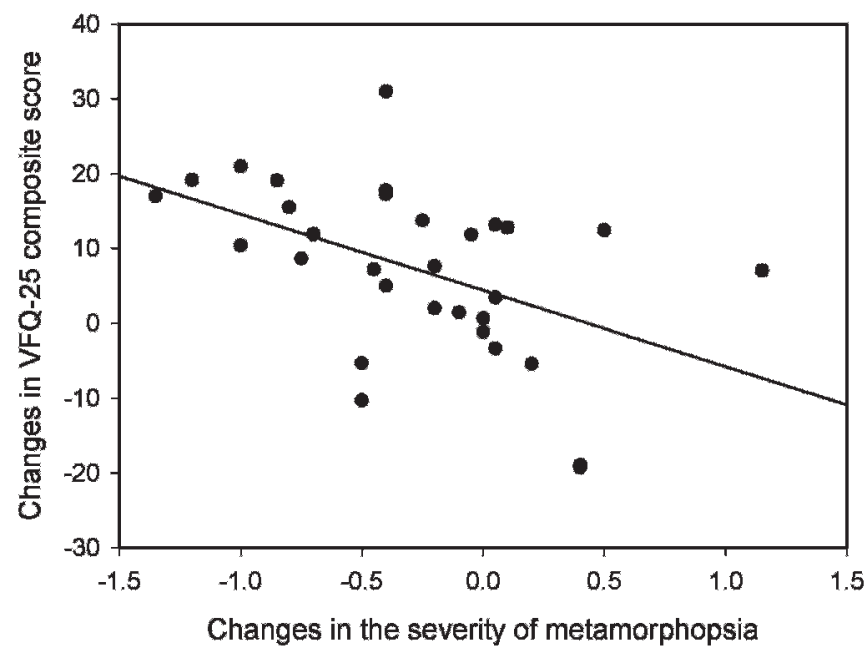

Figure 1 Statistically significant correlation between changes in the severity of metamorphopsia and changes in the 25-item National Eye Institute Visual Function Questionnaire (VFO-25) composite score by vitrectomy for macular hole ( $\mathrm{p}<0.01$, multiple regression analysis).

multiple regression analysis in the current study revealed that the severity of metamorphopsia was the single factor relevant to VR-QOL in patients with $\mathrm{MH}$, while visual acuity was not included among the relevant factors. Moreover, the presence and severity of cataract were not associated with VR-QOL. Thus, metamorphopsia plays a key role in the deterioration of visual functioning and VR-QOL in patients with $\mathrm{MH}$, even after successful closure of $\mathrm{MH}$ by vitrectomy. The severity of metamorphopsia after vitrectomy was actually 0.45 (SD 0.44), which is abnormally worse compared with normal controls. ${ }^{89}$

We found no association between letter contrast sensitivity and VFQ-25 composite score before and after surgery. GhaziNouri et al reported that VFQ-25 responses correlated with visual acuity but not with contrast sensitivity obtained with the Pelli-Robson chart in patients with epiretinal membrane. ${ }^{17}$ In patients with proliferative diabetic retinopathy, VFO-25 composite scores showed significant correlations with letter

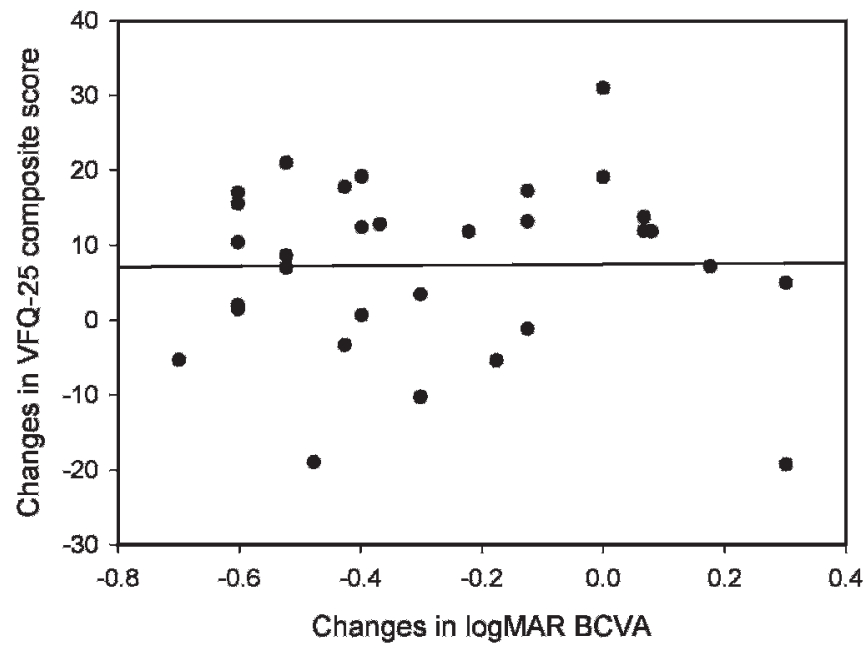

Figure 2 No significant correlation was found between changes in logarithm of minimum angle of resolution (logMAR) best corrected visual acuity (BCVA) and changes in the 25-item National Eye Institute Visual Function Questionnaire (VFO-25) composite score by vitrectomy for macular hole ( $p>0.05$, multiple regression analysis). 


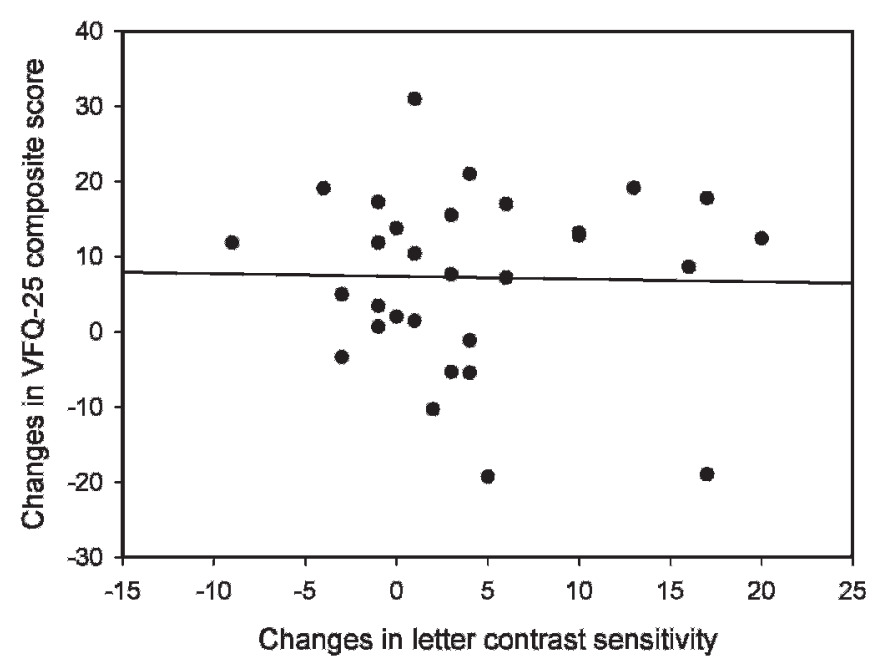

Figure 3 No significant correlation was found between changes in letter contrast sensitivity and changes in the 25-item National Eye Institute Visual Function Questionnaire (VFO-25) composite score by vitrectomy for macular hole ( $p>0.05$, multiple regression analysis).

contrast sensitivity, as well as visual acuity. ${ }^{18}$ In patients after retinal reattachment surgery, VFQ-25 composite score was significantly correlated with contrast sensitivity and lowcontrast visual acuity, whereas there was no correlation between the VFQ-25 composite score and BCVA. ${ }^{19}$ In a heterogeneous group of patients with multiple sclerosis,
VFQ-25 composite scores were found to be modestly and significantly correlated with several clinical parameters, including visual acuity, contrast sensitivity, visual field, and 100-Hue colour vision. ${ }^{20}$ In patients with Bothnia dystrophy, a defined type of retinitis pigmentosa, age and low contrast visual acuity were significantly associated with the NEI-VFQ-25 composite score. ${ }^{21}$ Thus, factors contributing to VR-QOL are not uniform across different type of ocular diseases, and therefore it seems worthwhile to investigate individual diseases from the view point of VR-QOL.

We acknowledge certain limitations to our study. First, the sample size in our study was rather small. Second, postoperative follow-up was short. A previous study showed that VFQ-25 in patients with $\mathrm{MH}$ was more improved at 1 year postoperatively than at 3 months postoperatively. ${ }^{3}$ Moreover, metamorphopsia might have further improved with a longer follow-up. Tranos et al reported, however, that $65 \%$ of patients complained of metamorphopsia at 4 months after surgery. ${ }^{4}$ Future studies with a larger sample size and longer follow-up time will further facilitate our understanding of the relationship between VR$\mathrm{QOL}$ and visual function, and other factors, in patients undergoing vitrectomy for $\mathrm{MH}$.

In conclusion, the current study quantitatively indicated that $\mathrm{MH}$ surgery significantly improved VR-QOL, which was dependent on the improvement of metamorphopsia. The severity of preoperative and postoperative metamorphopsia was significantly correlated with preoperative and postoperative VFO-25 scores, respectively. Metamorphopsia is an important factor not only in visual functioning, but also in the subjective

Table 3 Results of multiple regression analysis on the National Eye Institute 25-item Visual Function Questionnaire (VFO-25) composite score and explanatory variables

\begin{tabular}{|c|c|c|c|}
\hline Variable & $\beta$ & SE & p Value \\
\hline \multicolumn{4}{|l|}{ Preoperative VFQ- 25 composite score (objective variable) } \\
\hline \multicolumn{4}{|l|}{ Explanatory variable } \\
\hline LogMAR BCVA in the better-seeing eye & -0.173 & 8.910 & 0.419 \\
\hline LogMAR BCVA in the worse-seeing eye & 0.020 & 6.704 & 0.933 \\
\hline Letter contrast sensitivity in the better-seeing eye & 0.236 & 3.132 & 0.332 \\
\hline Letter contrast sensitivity in the worse-seeing eye & -0.073 & 0.390 & 0.753 \\
\hline Metamorphopsia & -0.479 & 4.348 & $0.019^{*}$ \\
\hline Macular hole index & -0.252 & 4.896 & 0.249 \\
\hline Lens nuclear colour & 0.183 & 13.997 & 0.614 \\
\hline Lens nuclear opalescence & 0.163 & 4.066 & 0.567 \\
\hline Lens cortical opacity & 0.131 & 2.619 & 0.535 \\
\hline Lens posterior subcapsular opacity & -0.301 & 7.559 & 0.199 \\
\hline \multicolumn{4}{|l|}{ Postoperative VFQ-25 composite score (objective variable) } \\
\hline \multicolumn{4}{|l|}{ Explanatory variable } \\
\hline LogMAR BCVA in the better-seeing eye & -0.360 & 8.996 & 0.1270 \\
\hline LogMAR BCVA in the worse-seeing eye & -0.217 & 8.333 & 0.385 \\
\hline Letter contrast sensitivity in the better-seeing eye & -0.126 & 3.611 & 0.672 \\
\hline Letter contrast sensitivity in the worse-seeing eye & -0.254 & 0.848 & 0.277 \\
\hline Metamorphopsia & -0.563 & 7.228 & $0.049^{*}$ \\
\hline Macular hole index & 0.117 & 5.306 & 0.636 \\
\hline Lens nuclear colour & -0.069 & 14.587 & 0.849 \\
\hline Lens nuclear opalescence & 0.045 & 4.563 & 0.893 \\
\hline Lens cortical opacity & 0.240 & 2.600 & 0.280 \\
\hline Lens posterior subcapsular opacity & -0.238 & 7.463 & 0.329 \\
\hline \multicolumn{4}{|l|}{ Changes in the VFO-25 composite score (objective variable) } \\
\hline \multicolumn{4}{|l|}{ Explanatory variable } \\
\hline Changes in logMAR BCVA & -0.151 & 7.274 & 0.402 \\
\hline Changes in letter contrast sensitivity & -0.087 & 0.291 & 0.621 \\
\hline Changes in metamorphopsia & -0.594 & 4.177 & $0.002^{*}$ \\
\hline
\end{tabular}

$\beta$, standard regression coefficient; BCVA, best corrected visual acuity; SE, standard error.

${ }^{*}$ Significant at $\mathrm{p}<0.05$. 
QOL in patients with $\mathrm{MH}$, both preoperatively and postoperatively.

Funding: Supported in part by Grants-in-Aid 19390439 for Scientific Research from the Ministry of Education, Culture, Sports, Science and Technology, Japan.

Competing interests: None declared.

Ethics approval: The research followed the tenets of the Declaration of Helsinki. The study protocol was approved by the Institutional Review Board of Tsukuba University Hospital.

Patient consent: Obtained

Provenance and peer review: Not commissioned; externally peer reviewed.

\section{REFERENCES}

1. Aaberg TM, Blair CJ, Gass JD. Macular holes. Am J Ophthalmol 1970;69:555-62.

2. Kelly NE, Wendel RT. Vitreous surgery for idiopathic macular holes. Results of a pilot study. Arch Ophthalmol 1991;109:654-9.

3. Hirneiß C, Neubauer AS, Gass CA, et al. Visual quality of life after macular hole surgery: Outcome and predictive factors. Br J Ophthalmol 2007:91:481-4.

4. Tranos PG, Ghazi-Nouri SM, Rubin GS, et al. Visual function and subjective perception of visual ability after macular hole surgery. Am J Ophthalmol 2004;138:995-1002.

5. Tranos PG, Peter NM, Nath R, et al. Macular hole surgery without prone positioning. Eye 2007;21:802-6.

6. Amsler M. Quantitative vision. Trans Ophthalmol Soc UK 1949;69:397-410.

7. Amsler M. Earliest symptoms of disease of macular. Br J Ophthalmol 1953;37:521-37.

8. Matsumoto C, Arimura E, Okuyama S, et al. Quantification of metamorphopsia in patients with epiretinal membranes. Invest Ophthalmol Vis Sci 2003:44:4012-6.

9. Arimura E, Matsumoto C, Okuyama S, et al. Retinal contraction and metamorphopsia scores in eyes with idiopathic epiretinal membrane. Invest Ophthalmol Vis Sci 2005;46:2961-6.
10. Kusuhara S, Teraoka Escaño MF, Fujii S, et al. Prediction of postoperative visual outcome based on hole configuration by optical coherence tomography in eyes with idiopathic macular holes. Am J Ophthalmol 2004;138:709-16.

11. Chylack LT Jr, Wolfe JK, Singer DM, et al. The Lens Opacities Classification System III. The Longitudinal Study of Cataract Study Group. Arch Ophthalmol 1993;111:831-6.

12. Mangione $\mathbf{C M}$, Lee PP, Gutierrez PR, et al. National Eye Institute Visual Function Questionnaire Field Test Investigators. Development of the 25-item National Eye Institute Visual Function Questionnaire. Arch Ophthalmol 2001;119:1050-8.

13. Suzukamo Y, Oshika T, Yuzawa M, et al. Psychometric properties of the 25-item National Eye Institute Visual Function Questionnaire (NEI VFO-25), Japanese version. Health Qual Life Outcomes 2005;3:65.

14. Miskala PH, Bass EB, Bressler NM, et al. Surgery for subfoveal choroidal neovascularization in age-related macular degeneration: quality-of-life findings: SST report no. 12. Ophthalmology 2004;111:1981-92.

15. Deramo VA, Cox TA, Syed AB, et al. Vision-related quality of life in people with central retinal vein occlusion using the 25-item National Eye Institute Visual Function Questionnaire. Arch Ophthalmol 2003;121:1297-1302.

16. Hyman LG, Komaroff E, Heijl A, Bengtsson B, Leske MC, Early Manifest Glaucoma Trial Group. Treatment and vision-related quality of life in the early manifest glaucoma trial. Ophthalmology 2005;112:1505-13.

17. Ghazi-Nouri SM, Tranos PG, Rubin GS, et al. Visual function and quality of life following vitrectomy and epiretinal membrane peel surgery. Br J Ophthalmol 2006;90:559-62.

18. Okamoto F, Okamoto Y, Fukuda S, et al. Vision-related quality of life and visual function following vitrectomy for proliferative diabetic retinopathy. Am J Ophthalmol 2008;145:1031-6.

19. Okamoto $\mathbf{F}$, Okamoto $Y$, Hiraoka $T$, et al. Vision-related quality of life and visual function after retinal detachment surgery. Am J Ophthalmol 2008;146:85-90.

20. Noble J, Forooghian F, Sproule M, et al. Utility of the National Eye Institute VFO-25 questionnaire in a heterogeneous group of multiple sclerosis patients. Am J Ophthalmol 2006;142:464-8.

21. Burstedt MS, Mönestam E, Sandgren O. Associations between specific measures of vision and vision-related quality of life in patients with bothnia dystrophy, a defined type of retinitis pigmentosa. Retina 2005;25:317-23. 